\title{
A NOTE ON FUNCTION QUANTIFICATION
}

\author{
J. W. ADDISON ${ }^{1}$ AND S. C. KLEENE
}

In $[7$, p. 211] the question was raised whether, for $k>0$, each predicate expressible in both the $k+1$-function-quantifier forms is hyperarithmetical in predicates expressible in the $k$-function-quantifier forms. In this note we answer this question in the negative, ${ }^{2}$ and use our answer in comparing the hierarchies $\mathfrak{N}_{0}, \mathfrak{N}_{1}, \mathfrak{N}_{2}, \cdots$ and $\Omega_{0}, \Omega_{1}, \Omega_{2}, \cdots$ of $[7$, XXIX].

The key to the question is that the following analogs of $[5,(5)$ and (6)] hold:

$$
\begin{gathered}
(\alpha)(E x)(E \beta) A(\bar{\alpha}(x), \beta) \equiv(E \beta)(\alpha)(E x) A\left(\bar{\alpha}(x), \lambda t \beta\left(\overline{2}^{\alpha(x)} 3^{t}\right)\right), \\
(E \alpha)(x)(\beta) A(\bar{\alpha}(x), \beta) \equiv(\beta)(E \alpha)(x) A\left(\bar{\alpha}(x), \lambda t \beta\left(2^{\bar{\alpha}(x)} 3^{t}\right)\right) .
\end{gathered}
$$

To prove (1), assume $(\alpha)(E x)(E \beta) A(\bar{\alpha}(x), \beta)$. For each $s$ such that $(E \beta) A(s, \beta)$, let $\beta_{s}$ be such a $\beta$; and for all other numbers $s$ let $\beta_{s}=\lambda t 0$. Then $\lambda u \beta_{(u)_{0}}\left((u)_{1}\right)$ is a function $\beta$ such that $(\alpha)(E x) A(\bar{\alpha}(x)$, $\left.\lambda t \beta\left(2^{\bar{\alpha}(x)} 3^{t}\right)\right)$. So we have the implication from left to right. The converse implication is immediate. (Formula (2) follows from (1) by duality, as (6) from (5) in [5].)

We can apply (1) to express $\mathfrak{R}_{2}(a)$ in both 2 -function-quantifier forms, as follows. Writing $\lambda_{1}$ for the representing function of $\Omega_{1}$, $\mathfrak{R}_{2}(a) \equiv(\alpha)(E x) T_{1}^{\mathfrak{R}_{1}, \alpha}(a, \quad a, \quad x) \equiv(\alpha)(E x) T_{1}^{1,1}\left(\bar{\lambda}_{1}(x), \quad \bar{\alpha}(x), \quad a, \quad a\right)$ $\equiv(\alpha)(E x) T_{1}^{1,1}\left(\bar{\lambda}_{1}(\operatorname{lh}(\bar{\alpha}(x))), \bar{\alpha}(x), a, a\right)$. Since $T_{1}^{1,1}\left(\bar{\lambda}_{1}(\operatorname{lh}(s)), s, a, a\right)$ is recursive in the 1 -function-quantifier predicate $\mathfrak{l}_{1}$, by $[7, \mathrm{XX}]$ it is expressible in both 2 -function-quantifier forms. Now use these two forms in the last expression for $\ell_{2}(a)$, apply (1) in the case of the form with existence first, and finally apply [5, Step 4, p. 316].

But $\Omega_{1}$ is of maximal hyperdegree for 1 -function-quantifier predicates, and $\mathfrak{R}_{2}$ is of greater hyperdegree than $\mathfrak{R}_{1}$ (cf. $[7, \mathrm{XXIX]}$ ). So

Presented to the Society, February 23, 1957; received by the editors January 12, 1957.

1 National Science Foundation Postdoctoral Fellow.

2 We first discovered this by adapting the proof of the invariance of the projective classes (other than $C A$ ) under the operation $(A)$ given in Kuratowski [8, $\$ 34, \mathrm{IX}]$. The proof we give here, using (1), is more direct; and indeed (1) yields a slightly more direct proof of the classical theorem than either Kuratowski's or the simpler but less clearly presented proof of Kantorovitch and Livenson [4].3 Analogies between the present theory and the hierarchies of descriptive set theory form the topic of $[1$, Part II], [2], and [3]; but this is the first instance of their use heuristically in the present series of papers (the material of $[5 ; 6 ; 7]$, and $[10]$ was discovered independently). 
$\mathfrak{R}_{\mathbf{2}}$ is not hyperarithmetical in 1 -function-quantifier predicates.

Thus the question is answered in the negative for $k=1$. A negative answer for any $k \geqq 1$ is obtained by showing similarly that $(\alpha)(E x) T_{1}^{\mathfrak{N}_{k}, \alpha}(a, a, x)$ is expressible in both $k+1$-function-quantifier forms $\left(\mathfrak{N}_{1}(a) \equiv(\alpha)(E x) T_{1}^{\alpha}(a, a, x), \mathfrak{N}_{2}(a) \equiv(E \alpha)(\beta)(E x) T_{1}^{\alpha, \beta}(a, a, x)\right.$, $\cdots) .^{3}$

Since $\mathfrak{N}_{2}$ is expressible in only one of the 2-function-quantifier forms $[7, X V I I I]$, it is not hyperarithmetical in $\Omega_{2}$ (similarly to [7, XXVIII]); so in hyperdegree $\mathfrak{R}_{2}$ lies properly between $\mathfrak{R}_{1}$ and $\mathfrak{R}_{2}$ $[7, \mathrm{XXIX}]$.

In fact, not only $\mathfrak{R}_{2}$, but the entire finite $\mathfrak{R}$-hierarchy $\mathfrak{R}_{2}, \mathfrak{\Omega}_{3}, \mathfrak{R}_{4}, \ldots$ (after $\mathfrak{R}_{0}, \mathfrak{R}_{1}$ ) and its extension into the transfinite up through the constructive third number class (at least) lie properly between $\mathfrak{N}_{1}$ and $\mathfrak{N}_{2}$ in hyperdegree.

To formulate this precisely, we first define a system of notations for the ordinals of the first three constructive number classes, extending the system $S_{3}$ (cf. [7, p. 199] or [6, p. 408, O1-O5]). The predicates $a \in O$ and $a<_{o} b$ of $S_{3}$ we may now also write $a \in O_{1_{o}}$ and $a<_{1_{o}} b$ (and for $|a|$ finite, $a \in O_{0_{o}}$ and $a<_{0_{o}} b$ ); and the corresponding predicates for the extended system $a \in O_{2_{o}}$ and $a<_{2_{o}} b$. The latter are defined by an inductive definition of the same form as the former, except for there being an additional direct clause: If $(b)[b \in O$ $\rightarrow\left\{\Phi_{1}(z, b)\right.$ is defined and $\left.\left.\in O_{2_{o}}\right\}\right]$ and $(b)(c)\left[b<_{o} c \rightarrow \Phi_{1}(z, b)\right.$ $\left.<_{2_{o}} \Phi_{1}(z, c)\right]$, then $3^{2} 5^{z} \in O_{2_{O}}$ and $(b)\left[b \in O \rightarrow \Phi_{1}(z, b)<_{2_{o}} 3^{2} 5^{z}\right]$.

Now our extension of the $\Omega_{k}$-hierarchy (for $k$ a natural number) is the $\mathfrak{S}_{y}$-hierarchy (for $y \in O_{2_{0}}$ ) which is established by the following definition, analogous to that which establishes the $H_{y}$-hierarchy (for $y \in O_{1_{o}}$ ) as an extension of the $L_{k}$-hierarchy (for $k$ a natural number): $\mathfrak{S}_{1}(a) \equiv a=a$. If $y=2^{z}$ and $z \in O_{2_{o}}, \mathfrak{S}_{y}(a) \equiv(\alpha)(E x) T_{1}^{\mathfrak{S}_{z}, \alpha}(a, a, x)$. For $i=1,2:$ If $y=3^{i} 5^{z}$ and $y \in O_{i_{o}}$,

$$
\mathfrak{S}_{y}(a) \equiv\left\{\begin{array}{l}
\mathfrak{S}_{\Phi_{1}\left(z,(a)_{1}\right)}\left((a)_{0}\right) \text { if }(a)_{1} \in O_{(i-1)_{o}}, \\
a=a \text { otherwise. }
\end{array}\right.
$$

To show that, for $y \in O_{2_{o}}, \mathfrak{S}_{y}$ is of lower hyperdegree than $\mathfrak{R}_{2}$, it will suffice to show that it is expressible in both 2-function-quantifier forms. We can do this by the technique used in [5, §8]. Accordingly we show that there is a primitive recursive function $\tau(y)$ such that, for $y \in O_{2_{O}}$,

${ }^{3}$ An alternative proof, adapted from Kantorovitch and Livenson [4],2 uses in place of (1) the formula $(\alpha)(E x) A(\bar{\alpha}(x)) \equiv\left(E_{\alpha}\right)_{\alpha \in Q}(x) A(\alpha(x))$ where $Q=\hat{\alpha}(\beta)(E i)(E j)$ $[\bar{\beta}(i)=\alpha(j)]$. 


$$
\begin{aligned}
\mathfrak{S}_{y}(a) & \equiv(E \alpha)(\beta)(E x) T_{1}^{\alpha, \beta}\left((\tau(y))_{0}, a, x\right) \\
& \equiv(\alpha)(E \beta)(x) \bar{T}_{1}^{\alpha, \beta}\left((\tau(y))_{1}, a, x\right) .
\end{aligned}
$$

In Case 2 of the proof (as modelled on that of [5, Theorem 9]), $y=2^{z}$ and $z \in O_{2_{o}}$. By the hypothesis of the induction,

$$
\begin{aligned}
\mathfrak{S}_{z}(a) & \equiv(E \alpha)(\beta)(E x) T_{1}^{\alpha, \beta}\left((\tau(z))_{0}, a, x\right) \\
& \equiv(\alpha)(E \beta)(x) \bar{T}_{1}^{\alpha, \beta}\left((\tau(z))_{1}, a, x\right) .
\end{aligned}
$$

By definition,

$$
\mathfrak{S}_{y}(a) \equiv(\alpha)(E x) T_{1}^{\mathfrak{W}_{2}, \alpha}(a, a, x) \equiv(\alpha)(E x) T_{1}^{\mathfrak{W}_{2}, 1}(\bar{\alpha}(x), a, a)
$$

(cf. [7, p. 209, 1. 12 from below]). Consider $T_{1}^{\mathfrak{Q}_{2}, 1}(s, a, a)$; if in the right side of (4) we first replace $\tau(z)$ by a variable $u$, and then apply the method of proof of [5, Theorem 5] using the resulting 2-functionquantifier predicates in place of $\mathfrak{S}_{z}$, we obtain primitive recursive predicates $R$ and $S$ such that

$$
\begin{aligned}
T_{1}^{\mathfrak{W}_{s, 1}}(s, a, a) & \equiv(E \beta)(\gamma)(E w) R(u, s, a, \beta, \gamma, w) \\
& \equiv(\beta)(E \gamma)(w) S(u, s, a, \beta, \gamma, w)
\end{aligned}
$$

when $u=\tau(z)$. Using these two expressions in place of $T_{1}^{\Phi_{z}, 1}(s, a, a)$ in (5), and applying (1) in the case of the first, and then [5, Step 4, p. 316], we obtain primitive recursive predicates $R_{2}$ and $S_{2}$ such that

$$
\begin{aligned}
\mathfrak{S}_{y}(a) & \equiv(E \alpha)(\beta)(E x) R_{2}(u, a, \alpha, \beta, x) \\
& \equiv(\alpha)(E \beta)(x) S_{2}(u, a, \alpha, \beta, x)
\end{aligned}
$$

when $u=\tau(z)$. Finally replacing $u$ in the right side of $(7)$ by $\Phi_{1}(t, z)$, and applying [5, Lemma 12] for two function quantifiers, we obtain primitive recursive functions $\phi_{2}$ and $\psi_{2}$ such that

$$
\begin{aligned}
\mathfrak{S}_{y}(a) & \equiv(E \alpha)(\beta)(E x) T_{1}^{\alpha, \beta}\left(\phi_{2}(t, z), a, x\right) \\
& \equiv(\alpha)(E \beta)(x) \bar{T}_{1}^{\alpha, \beta}\left(\psi_{2}(t, z), a, x\right)
\end{aligned}
$$

when $t$ is a Gödel number of $\tau$. So for Case 2 it will suffice to take $\tau(y)=2^{\phi_{2}\left(t,(y)_{0}\right)} \cdot 3^{\psi_{2}\left(t,(y)_{0}\right)}$ for $t$ a Gödel number of $\tau$.

In Case $4, y=3^{2} 5^{z}$ and $y \in O_{2_{0}}$. By the hypothesis of the induction, for each $b \in O$,

$$
\begin{aligned}
\mathfrak{S}_{\Phi_{1}(z, b)}(a) & \equiv(E \alpha)(\beta)(E x) T_{1}^{\alpha, \beta}\left(\left(\tau\left(\Phi_{1}(z, b)\right)\right)_{0}, a, x\right) \\
& \equiv(\alpha)(E \beta)(x) \bar{T}_{1}^{\alpha, \beta}\left(\left(\tau\left(\Phi_{1}(z, b)\right)\right)_{1}, a, x\right) .
\end{aligned}
$$

By definition, 


$$
\mathfrak{S}_{y}(a) \equiv\left[\mathfrak{S}_{\Phi_{1}\left(z,(a)_{1}\right)}\left((a)_{0}\right) \&(a)_{1} \in O\right] \vee(a)_{1} \notin O,
$$

where $\Phi_{1}\left(z,(a)_{1}\right)$ and hence $\mathfrak{E}_{\Phi_{1}\left(z,(a)_{1}\right)}\left((a)_{0}\right)$ may be undefined in the case that $(a)_{1} \notin O$, but then $\mathfrak{S}_{y}(a)$ is true anyway. Now if in the right side of (9) we first replace $\tau\left(\Phi_{1}(z, b)\right)$ by $\Phi_{1}\left(t, \Phi_{1}\left(z,(a)_{1}\right)\right)$, and $a$ by $(a)_{0}$, and then apply $[5$, Lemma 12$]$, we obtain primitive recursive predicates $R_{4}$ and $S_{4}$ such that

$$
\begin{aligned}
\mathfrak{S}_{\Phi_{1}\left(z,(a)_{1}\right)}\left((a)_{0}\right) & \equiv(E \alpha)(\beta)(E x) R_{4}(t, z, a, \alpha, \beta, x) \\
& \equiv(\alpha)(E \beta)(x) S_{4}(t, z, a, \alpha, \beta, x)
\end{aligned}
$$

when $(a)_{1} \in O$ and $t$ is a Gödel number of $\tau$. Replacing $\mathfrak{S}_{\Phi_{1}\left(z,(a)_{1}\right)}\left((a)_{0}\right)$ in the right side of (10) by the two 2-function-quantifier expressions of (11), and $(a)_{1} \in O$ by the 1 -function-quantifier expression given by $[7, \mathrm{XXV}]$ or [6, Theorem II], suitably advancing and contracting quantifiers (cf. $[5, \S 3]$ ), and applying [5, Lemma 12], we obtain primitive recursive functions $\phi_{4}$ and $\psi_{4}$ such that

$$
\begin{aligned}
\mathfrak{S}_{\nu}(a) & \equiv(E \alpha)(\beta)(E x) T_{1}^{\alpha, \beta}\left(\phi_{4}(t, z), a, x\right) \\
& \equiv(\alpha)(E \beta)(x) \bar{T}_{1}^{\alpha, \beta}\left(\psi_{4}(t, z), a, x\right)
\end{aligned}
$$

when $t$ is a Gödel number of $\tau$. So for Case 4 it will suffice to take $\tau(y)=2^{\phi_{4}\left(t,(y)_{2}\right)} \cdot 3^{\psi_{4}\left(t,(y)_{2}\right)}$ for $t$ a Gödel number of $\tau$.

Cases 1 and 3 offer no further difficulties, and the recursion theorem is used to solve for a Gödel number $t$ of $\tau$ after combining the cases (cf. the proofs of Lemmas 3-5 in [5]).

In getting this result we have used a basic theorem about the constructive second number class [6, Theorem II], but only the definition of the constructive third number class. Further exploration of the situation is planned in connection with the investigation of the constructive higher number classes, and the study of the analogies with the hierarchies of point sets in analysis, ${ }^{4}$ both referred to in $[7, \mathrm{p}$. 212].

\section{REFERENCES}

1. J. W. Addison, On some points of the theory of recursive functions, Doctoral Dissertation, University of Wisconsin, 1954.

2. - Analogies in the Borel, Lusin, and Kleene hierarchies I, Bull. Amer. Math. Soc. Abstract 61-1-139.

4 In particular, a discussion is planned of the relationship between an extended $\mathfrak{\$}$-hierarchy and the effective $C$-hierarchy (corresponding to the hierarchy of $C$-sets introduced by Luzin), and of the effective $R$-sets (corresponding to the $R$-sets introduced by Kolmogorov) and of the effective analogs of still more general subclasses of $B_{2}$ (cf. [9]). 
3. - Analogies in the Borel, Lusin, and Kleene hierarchies II, Bull. Amer. Math. Soc. Abstract 61-2-341.

4. L. Kantorovitch and E. Livenson, Sur quelques théorèmes concernant la theorie des ensembles projectifs, C. R. Acad. Sci. Paris vol. 204 (1937) pp. 466-467.

5. S. C. Kleene, Arithmetical predicates and function quantifiers, Trans. Amer. Math. Soc. vol. 79 (1955) pp. 312-340. Errata, ibid. vol. 80 (1955) p. 386 (on p. 325 last line of text, the subscript of " $z$ " should be " $(t)_{0}$ "; on p. 3301.10 , the superscript " $H^{n}$ should be dotted); ibid. vol. 81 (1956) p. 524 ; on p. 333, supply a subscript " 1 " on " $T$ " in (20) and (21).

6. - On the forms of the predicates in the theory of constructive ordinals (second paper), Amer. J. Math. vol. 77 (1955) pp. 405-428.

7. - Hierarchies of number-theoretic predicates, Bull. Amer. Math. Soc. vol. 61 (1955) pp. 193-213. Errata: p. 2001.11 from below, for "30" read "29"; p. 2111.17 from below, replace dot by " $k$ ".

8. C. Kuratowski, Topologie I, 2d ed., Warszawa-Wrocław, 1948, XI +450 pp.

9. A. A. Lyapunov, R-mnozestva, Trudy. Mat. Inst. Steklov vol. 40 (1953) 68 pp.

10. C. Spector, Recursive well-orderings, J. Symbolic Logic vol. 20 (1955) pp. 151163.

INSTITUTE FOR ADVANCED STUdY AND

Princeton University 